\title{
Uric acid as a biomarker for pregnancy induced hypertension
}

\author{
Ajay Puri ${ }^{1}$, Jyoti Kohli ${ }^{2 *}$, Sapna Puri ${ }^{3}$ \\ ${ }^{1,2}$ Associate Professor, ${ }^{3}$ Professor, ${ }^{1}$ Dept. of Biochemistry, ${ }^{2}$ Dept. of Microbiology, ${ }^{3}$ Dept. of Gynaecology and Obstetrics, Acharya Shri \\ Chander College of Medical Sciences Jammu (ASCOMS), J\&K, India
}

\section{*Corresponding Author: Jyoti Kohli}

Email: puria062@gmail.com

\begin{abstract}
Background: Pregnancy induced hypertension is the most common complication of pregnancy. It affects 5-7\% of pregnant women. Pregnancy induced hypertension is characterized by vasospasm which is associated with platelet aggregation and reduced uteroplacental flow. In addition to these, altered renal function tests can also worsens the condition. This influences the maternal as well as fetal outcome. Aims: To prevent the complications of pregnancy induced hypertension and for proper management of these cases present study was conducted.

Materials and Methods: The present study was conducted in 300 subjects grouped into 120 cases of normal pregnancy, 150 cases of pregnancy induced hypertensives, 30 cases of eclamptics and serum uric acid level was measured in these.

Results and Discussion: elevated level of serum uric acid was seen with increase in gestation period and rise in diastolic blood pressure. The p- value was estimated by student t-test in different groups and was found to be $<0.001$ which was highly significant and correlated. Conclusion: Hence Serum Uric acid is the significant and reliable indicator for pregnancy induced hypertension to prevent the fetal and maternal mortality and morbidity.
\end{abstract}

Keywords: Eclampsia, Gestational hypertension, Hyperuricemia, Pre-eclampsia, Pregnancy induced hypertension(PIH).

\section{Introduction}

Hypertensive disorders are the commonest medical disorders during pregnancy which contributes to maternal and perinatal mortality and morbidity. Approximately 2$10 \%$ of pregnancies are complicated by hypertensive disorders. ${ }^{1}$ Hypertensive disorders of pregnancy included gestational hypertension and pre- eclampsia. ${ }^{2}$ Gestational hypertension is defined as a sustained rise of blood pressure to $140 / 90 \mathrm{~mm} \mathrm{Hg}$ or more on at least two occasions 4 or more hours apart beyond $20^{\text {th }}$ week of pregnancy or within first $48 \mathrm{hrs}$ of delivery in previously normotensive women whereas pre-eclampsia can be defined as multisystem disorder of unknown etiology characterized by development of hypertension to the extent of $140 / 90 \mathrm{~mm} \mathrm{Hg}$ or more with proteinuria after $20^{\text {th }}$ week in a previously normotensive and non- proteinuric women and eclampsia can be defined as when pre-eclampsia is associated with generalized tonicclonic seizures and or coma. ${ }^{3}$

Gestational hypertension is considered as a risk factor for cardiovascular disease and metabolic syndrome in nonpregnant females. ${ }^{4}$ Patients with gestational hypertension share some common risk factors and pathophysiological abnormalities such as increased maternal age, raised body mass index, and hyperuricemia. ${ }^{5}$ Pregnancy may induce hypertension in women who are normotensive or may precipitate hypertension in hypertensive mothers. ${ }^{6}$ In $1917-$ Slemons and Bogert first determined that the maternal serum concentration of uric acid is elevated in preeclampsia and eclampsia, complications which can result in severe morbidity and even mortality in pregnant females. Raised serum uric acid associated with pre-eclamptic pregnancies has been reported a century ago. ${ }^{7}$ Hyperuricemia is an independent predictor of cardiovascular and renal disease during pregnancy. ${ }^{8}$ Maternal serum uric acid level can be used as a biomarker for pre-eclampsia during pregnancy and serum uric acid level correspond to the severity of pre- eclampsia. Pre-eclampsia increase the risk of fetal death five fold and kills fifty thousand women a year. Women with pregnancy induced hypertension are found with increased level of uric acid in maternal blood due to decreased renal urate excretion. Reduced uric acid clearance may be secondary to decreased glomerular filteration rate and decreased secretion resulting hyperuricemia in patients with pregnancy induced hypertension..$^{9,10}$

\section{Materials and Methods}

The present study of two years of duration from September 2017 to September 2019 conducted in the department of Biochemistry in collaboration with department of Obstetrics and Gynaecology and Microbiology at Acharya Shree Chander College of Medical Sciences and Hospital Jammu. The present study divided in two groups of pregnant women- one with Pregnancy Induced Hypertension and other of healthy pregnant women. Blood pressure measurement and urine analysis was performed at the beginning of pregnancy to exclude pre- existing proteinuria or renal disease.

\section{Inclusion criteria}

Both groups were pregnancy of 24-36 gestational weeks. For pregnancy induced hypertension- a systolic blood pressure level of $140 \mathrm{~mm} \mathrm{Hg}$ or higher or a diastolic blood pressure level of $90 \mathrm{~mm} \mathrm{Hg}$ or higher that occurred after 20 weeks of gestation without proteinuria in $24 \mathrm{hrs}$ urine specimen.

\section{Exclusion criteria}

Non pregnant females, females suffering from chronic ailments and renal diseases. Serum uric acid levels were 
measured by enzymatic method in cobas C311 autoanalyser. In patients with PIH total urinary protein levels were measured by modification of dye binding method used by Fujita et al and commercial uric 3V SGO 3100. Pregnancy induced hypertension is divided into 3 groups on the basis of Blood Pressure measurement:

Mild PIH B.P is > $140 / 90 \mathrm{mmHg}$

Moderate PIH $<160 / 110 \mathrm{mmHg}$

Severe PIH > 160/110mmHg

But without convulsions

Eclamptic > 160/110mmHg

With convulsions

\section{Results}

In the present study total number of 300 cases were studied. These included the normal pregnancy of 120 cases which were divided into first, second and third trimester of 40 cases each and 150 cases of pregnancy induced hypertension. 150 cases of pregnancy induced hypertension were subdivided into 50 cases of mild $\mathrm{PIH}$, moderate $\mathrm{PIH}$, severe PIH each and also included 30 cases of eclamptic cases with history of convulsions and high blood pressure. Uric acid levels in different trimesters of pregnancy and different stages of pregnancy induced hypertension studied which was showed statistically in table 1 . The mean and standard deviation of uric acid levels estimated in different trimesters of pregnancy, in different stages of PIH, also in eclamptic cases and compared with each other. The p-value in different groups studied were estimated by using student $-\mathrm{t}$ test as shown in table 2 .

Table 1: Comparison of mean and SD Value of Uric acid in normal Pregnancy and PIH

\begin{tabular}{|l|c|c|c|c|}
\hline Types of cases & Mean & SD & $\begin{array}{c}\text { No. of } \\
\text { cases }\end{array}$ & Range \\
\hline First Trimester & 3.13 & 0.67 & 40 & $2.0-4.6$ \\
\hline $2^{\mathrm{ND}}$ Trimester & 2.09 & 0.88 & 40 & $1.0-4.0$ \\
\hline $3^{\mathrm{RD}}$ Trimester & 4.8 & 1.08 & 40 & $2.6-6.2$ \\
\hline Mild PIH & 6.9 & 0.75 & 50 & $5.4-8.0$ \\
\hline Moderate PIH & 7.8 & 0.83 & 50 & $6.0-9.0$ \\
\hline Severe PIH & 8.01 & 0.32 & 50 & $6.2-8.5$ \\
\hline Eclamptic & 9.03 & 0.40 & 30 & $7.5-9.5$ \\
\hline
\end{tabular}

Table 2: Comparison of $\mathrm{P}$ valves of different groups studied

\begin{tabular}{|l|c|c|}
\hline Different groups & P valve & Level of significance \\
\cline { 1 - 1 } First Trimester & $<0.0001$ & Highly significant \\
\cline { 1 - 1 } $2^{\text {nd }}$ Trimester & & \\
\cline { 1 - 1 } $2^{\text {nd }}$ Trimester & $<0.0001$ & Highly significant \\
\cline { 1 - 1 } $3^{\text {rd }}$ Trimester & $<0.0001$ & Highly significant \\
\cline { 1 - 1 } $3^{\text {rd }}$ Trimester & $<0.0001$ & Highly significant \\
\cline { 1 - 1 } Mild PIH & $<0.0001$ & Highly significant \\
\cline { 1 - 1 } $2^{\text {nd }}$ Trimester & & \\
\cline { 1 - 1 } Mild PIH & $<0.098$ & Non significant \\
\cline { 1 - 1 } Mild PIH & & \\
\cline { 1 - 1 } Moderate PIH & &
\end{tabular}

\begin{tabular}{|l|c|c|}
\hline $2^{\text {nd }}$ Trimester & $<0.0001$ & Highly significant \\
\cline { 1 - 1 } Moderate PIH & & \\
\cline { 1 - 1 } $2^{\text {nd }}$ Trimester & $<0.0001$ & Highly significant \\
\cline { 1 - 1 } Severe PIH & $<0.0001$ & Highly significant \\
\cline { 1 - 1 } $2^{\text {nd }}$ Trimester & $<0.0001$ & Highly significant \\
\cline { 1 - 1 } Eclamptic & & \\
\cline { 1 - 1 } Severe PIH & & \\
\cline { 1 - 1 } Eclamptic & &
\end{tabular}

\section{Discussion}

In the present study table 1 showed the different ranges of uric acid level in different groups of pregnancy and also in pre-eclamptic and eclamptic stages. It varies from minimum value of 2.0 to maximum value of 9.5 in different stages of normal pregnancy to pregnancy induced hypertension. This showed that serum uric acid level slowly decreases in normotensive pregnancy until 16 weeks of gestation due to expansion of plasma volume, increased renal clearance and the uricosuric effect of estrogen. During $2^{\text {nd }}$ trimester the uric acid level remain stable and increases during $3^{\text {rd }}$ trimester due to increase production of uric acid. ${ }^{11}$ The pregnant with history of pre-eclampsia are more prone to cardiovascular disorders and hypertension later on. ${ }^{12,13}$ It was observed in the present study that serum uric acid level rises in pregnancy induced hypertension and eclampsia. It also showed that uric acid level correlates with plasma rennin activity. ${ }^{14}$ The mean and standard deviation of uric acid estimated in different trimesters of normal pregnancy were $3.13 \pm 0.67$ in $1^{\text {st }}$ trimester, $2.09 \pm 0.88$ in $2^{\text {nd }}$ trimester, $4.8 \pm 1.08$ where as the mean and standard deviation of uric acid estimated in different stages of pregnancy induced hypertension were $6.9 \pm 0.75$ in mild $\mathrm{PIH}, 7.8 \pm 0.83$ in moderate PIH, $8.01 \pm 0.32$ in severe PIH and 9.03+_0.40 in eclamptic cases as shown in table 1 whereas the study done by Kondareddy et $\mathrm{al}^{15}$ also correlates with the present study in which mean and standard deviation of uric acid level in normotensives was $3.52 \pm 0.23$, mild pre-eclampsia it was $5.50+\_0.34$, in severe pre-eclampsia it was $6.25 \pm 0.33$ and in eclampsia it was $7.43 \pm 1.16$. Table No. II showed the pvalues of different studied groups which were highly significant in all the groups studied i.e. $<0.001$ except in moderate to severe PIH where it was non significant. Thus uric acid was a significant predictor of pregnancy induced hypertension with $\mathrm{p}$-value $<0.001$ which was highly significant and correlates with the study done by Prakash et al, ${ }^{16}$ Gianni Bellomo, et al, ${ }^{17}$ Hawkins et al, ${ }^{18}$ Tejal, et al. ${ }^{19}$ Although numerous studies have been conducted to find out the relationship between elevated uric acid level and preeclampsia. ${ }^{20}$ The uric acid is a product of purine metabolism. Most of the purines are synthesized in liver but some come directly from food. $2 / 3^{\text {rd }}$ of uric acid is excreted in kidneys and remainder via gastrointestinal tract. In the kidneys uric acid undergoes both reabsorption and secretion but there is net reabsorption in the renal tubules $(88-93 \%$ of filtered load). In normal pregnancy the serum uric acid concentration is $25-35 \%$ lower than non- pregnant levels during most of the pregnancy. Toward the end of pregnancy 
the level rises towards normal. The mechanism involved is increased glomerular filteration during pregnancy and decreased fractional reabsorption of uric acid in the proximal renal tubules during pregnancy. Defective second stage of trophoblastic invasion in the placenta and production of an ischemic metabolite. That ischemic metabolite causes peripheral vasoconstriction in systemic vessels, glomeruli, liver, central nervous system, eyes. Glomerular vasoconstriction and capillary bed damages (glomerular endotheliosis). Reduced glomerular filteration rate and increased proximal tubular uric acid net reabsorption leading to raised serum levels. Other less significant mechanism of increased uric acid tubular reabsorption are increased sympathetic activity, suppressed angiotensin system activity and reduced estrogen level. ${ }^{21}$ Some have argued that raised serum lactate levels in preeclampsia also decreases tubular secretion of uric acid. Studies have used probencid which reduces tubular reabsorption is the predominant mechanism of elevated uric acid level in pre-eclampsia. Besides reduced renal clearance, increased production of uric acid secondary to placental ischemia resulting in fetal hypoxia due to increased serum level of purine metabolites. ${ }^{22}$ These metabolites can cross the maternal circulation which are degraded by maternal xanthine oxidase and there is increase level of uric acid and fetal growth retardation seen.

\section{Conclusion}

Hyperuricemia also predicts the development of hypertension. ${ }^{23}$ In the present study positive correlation is seen between the increase in serum uric acid level and diastolic blood pressure. Plasma uric acid is a better indicator than blood pressure for fetal outcome. ${ }^{24}$ Hence forth the serum uric acid level detection in nomotensives in normal pregnancy as well as in pregnancy induced hypertensives and eclamptics can prevent the complications. This can also help in the management of these cases to avert the maternal and fetal outcome.

\section{Source of Funding}

None.

\section{Conflict of Interest}

None.

\section{References}

1. Tejal P, Astha D. Relationship of serum uric acid level to maternal and perinatal outcomes in patients with hypertensive disorders of pregnancy. Gujarat Med J. 2014;69(2):47-52.

2. Nirmala A, Lamsal M, Majhi S, Khan SA, Basnet P. Significance of uric acid in pregnancy induced hypertension. $J$ Natl Med Assoc. 2017;109(3):198-202.

3. Dutta DC. Hypertensive disorders in pregnancy. D.C. Dutta text book of obstetrics $8^{\text {th }}$ edition: 255-63.

4. Ponta A, Hartikainen A, Sovio U. Manifestations of metabolic syndrome after hypertensive pregnancy; 2004.

5. Stella LC, Brien O, Forrester MJ. The co existence of gestational hypertension and diabetes- influence on pregnancy outcome. Am J Perinatol. 2008;25(6):325-9.
6. Conde- Agudelo, A and Belizh, JM. Risk factors for pre eclampsia in a large cohort of Latin sAmerican and Carribean Women. BJOG. 2000;107(1):75-83.

7. Siemons JM and Bogert L. The uric acid content of maternal and fetal blood. J Biol Chem. 1917;32(1).

8. Patel. T and Dudhat A. Relationship of serum uric acid level to maternal and perinatal outcome in patients with hypertensive disorders of pregnancy. Gujarat Med J. 2014;69(2):1-3.

9. Siemons JM and Bogert L. The uric acid content of maternal and fetal blood. J Biol Chem. 1917;32(1):63-9.

10. Powers RW, Boduas LM, Ness RB. Uric acid concentrations in early pregnancy among pre- eclamptic women with gestational hyperuricemia at delivery. Am J Obstet Gynecol. 2006;194(1):160.

11. Mervil P, Bar, Beaufils M. Lone Hyperuricemia during pregnancy, maternal and fetal out comes. Euro. J Obstet gynecol Reprod Bio. 1998;177:145-50.

12. Irgens HA, Reisaeter L, Irgens LM. Long term mortality of mothers and fathers after pre- eclampsia. Population based cohort study. BMJ. 2001;323:1213-7.

13. Sibai BM, el- Nazer A, Gonzalez- Ruiz A. Severe preeclampsia- eclampsia in young primigravid women. Subsequent pregnancy outcome and remote prognosis. Am J Obstet Gynecol. 1986;155:1011-6.

14. Mazzali M, Hughas J, Kim YG. Elevated uric acid increases blood pressure in the rat by a novel crystal independent mechanism. Hypertens. 2001;38: 1101-6.

15. Reddy TK, Prathap T. Uric acid as an important biomarker in hypertensive disorders in pregnancy. Int J Reprod contracept Obstet Gynecol. 2016;5(12):4382-4.

16. Prakash S, Sharma N, Kumari P, Kumar A. Serum uric acid as marker for diagnosing pre-eclampsia. IJPSR. 2012;3(8):266975 .

17. Bellomo G, Venanzi S, Saronio P. Prognostic significance of uric acid in gestational hypertension. Hypertens. 2011;58(4):704-8.

18. Hawkins AT, Roberts JM, Mangos GJ, Davis, Roberts LM, Brown HA. Plasma uric acid remains a marker of poor out come in hypertensive pregnancy. A retrospective cohorts study. BJOG. 2012;119:484-92.

19. Tejal P, Astha D. Relationship of serum uric acid level to maternal and perinatal out come in patients with hypertensive disorders of pregnancy. Gujarat Med J. 2014;69:45-7.

20. Cotter AM, Martin CM, Leary J, Daly SF. Increased fetal DNA in maternal circulation in early pregnancy is associated with an increased risk of pre- eclampsia. Am J Obstet Gynecol. 2004;191(2):515-20.

21. Ferris TF, Gorden P. Effect of angiotensin and norepinephrine upon urate clearance in man. Am J Med. 1968;44:359-65.

22. Saugstad OD. Hypoxanthine as measurement of hypoxia. Pediatr Res. 1975;9:158-61.

23. Johnson RJ, Kang DH, Feig D. Is there a pathogenetic role for uric acid in hypertension and cardiovascular and renal disease. Hypertens. 2003;41:1183-90.

24. Redman CW, Beillin LJ, Bonnar J, Wilkinson RH. Plasma urate measurement in predicting fetal death in hypertensive pregnancy. Lancet. 1976;1:1370-3.

How to cite: Puri A, Kohli J, Puri S. Uric acid as a biomarker for pregnancy induced hypertension. Panacea $J$ Med Sci. 2020;10(1):51-3. 